\title{
Characteristics Analysis of the Flow Field of Self Balanced Multi-stage Centrifugal Pump
}

\author{
Jin-Gang LIU ${ }^{1}$, Yao YAO ${ }^{1, a,}$, , Quan $\mathrm{Li}^{2}$, and Jian-Wen $\mathrm{CHEN}^{3}$ \\ ${ }^{1}$ School of Mechanical Engineering, Xiangtan University, 411105 Xiangtan, China \\ ${ }^{2}$ School of mechanical and vehicle engineering, Hunan University,410000 Changsha,China \\ ${ }^{3}$ Jianglu Electromechanical Science \& Technology Co.,Ltd., 411105 Xiangtan,China \\ ayaowings@163.com
}

Keywords: Multi-stage centrifugal pump, Fluid analysis, FLUENT

\begin{abstract}
The flow field characteristics of self balanced multi-stage centrifugal pump were analyzed based on FLUENT. Reynolds-averaged Navier-Stokes equations and standard k- $\varepsilon$ model are adopted to calculate the pressure and velocity distribution of the flow field in the impeller and the guide vane under three different conditions. The results showed that the trend of head and efficiency calculated from the simulation is in agreement with the experimental values, and there is not much difference in the flow field distribution between the first stage and the fourth stage impeller and the guide vane. Thus, the found of this paper has certain guiding significance for the design and optimization of self balanced multi-stage centrifugal pump.
\end{abstract}

\section{Introduction}

Multi-stage centrifugal pumps are widely used in industrial, civil, building water supply and drainage, HVAC, refrigeration, sewage, fire, chemical, pharmaceutical and other fields, the internal flow characteristics directly affect the performance of multi-stage centrifugal pump. The axial force is an important factor that must be considered in the design and operation of multi stage pump. It will directly affect the safe and reliable operation of the pump[1].It is a common method of symmetrical arrange the impeller to balance the axial force, which make the majority of multi-stage pump axial force cancelled out, and greatly simplifying the structure of multi-stage pump and improve the efficiency $[2,4]$. With the development of computational fluid dynamics, Performance prediction and optimum design of centrifugal pump based on CFD has already met the project practical requirements [5].

This paper utilizes FLUENT to analyze the internal flow field of self balanced Multi-stage Centrifugal Pump.

\section{Calculation Model and Experimental Verification}

\section{Geometric Models}

The model of self balanced multi-stage centrifugal pump calculation build by UG, which shown in Figure 1.

The pump body is composed of six (even level) flow channels, with the discharge section on center, impeller positive and negative symmetrical arrangement. The fluid is sucked from the primary suction section (IN), enters the first stage impeller and the guide vane (1), and guided into the pipe from the third stage guide vane (2), then enters the fourth stage impeller and guide vanes(4) form the secondary suction section (3), and finally outflow from the discharge section (OUT). 


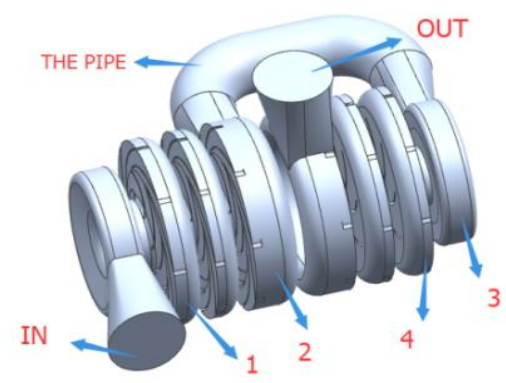

Figure1.Model of self-balanced multi-stage centrifugal pump

Self balanced multi-stage centrifugal pump model conditions and physical properties are shown in Table 1 below.

Table 1.working conditions and physical parameters

\begin{tabular}{|l|l|}
\hline Rated condition & $288\left(\mathrm{~m}^{3} / \mathrm{h}\right)$ \\
\hline Condition field & $3.75 \sim 850\left(\mathrm{~m}^{3} / \mathrm{h}\right)$ \\
\hline Rotational speed & $1480(\mathrm{r} / \mathrm{min})$ \\
\hline Working fluid & Water \\
\hline Fluid density & $998\left(\mathrm{~kg} / \mathrm{m}^{3}\right)$ \\
\hline Atmospheric pressure & $1.01 \times 10^{5}(\mathrm{~Pa})$ \\
\hline
\end{tabular}

\section{Meshing}

This model of self-balanced multi-stage centrifugal pump is put into ICEM software and carved up it into grids. In this paper, the 3D unstructured tetrahedral mesh are adopted. The whole centrifugal pump fluid region is calculated and the total number of units is 9264570, and the total number of nodes is 1621400 . The computational mesh is shown in Figure.2.

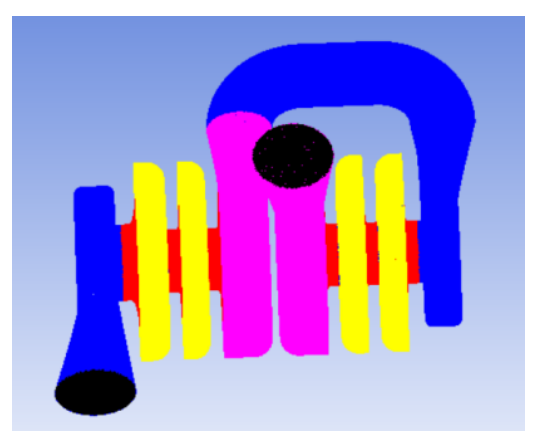

Figure2.Grid of Calculation Region

\section{Control Equation}

Continuity equation:

$$
\frac{\partial_{u}}{\partial_{x}}+\frac{\partial_{v}}{\partial_{y}}+\frac{\partial_{w}}{\partial_{z}}=0
$$

Where:

$\mathrm{u}, \mathrm{v}, \mathrm{w}$ is the velocity component of velocity in the space coordinate system

Momentum equation:

$$
\frac{\partial\left(\rho u_{i}\right)}{\partial_{t}}+\frac{\partial\left(\rho u_{i} u_{j}\right)}{\partial x_{i}}=\mu \frac{\partial^{2}}{\partial x_{i} \partial x_{j}}-\frac{\partial p}{\partial x_{i}}+\rho F_{i}
$$

Where: $u_{i}, u_{j}(\mathrm{i}, \mathrm{j}=1,2,3)$-velocity component, $x_{i}, x_{j}(\mathrm{i}, \mathrm{j}=1,2,3)$ - coordinate component, 
$\rho F_{i}$ - mass force, $\mathrm{u}$ - Viscosity, $\mathrm{P}$ - pressure[6,7].

\section{Calculation Model and the Setting of Boundary Conditions}

Based on software Fluent, assume that the centrifugal pump flow is constant and the internal flow field in the whole flow channel is a 3D incompressible steady turbulent flow field, operating reference pressure is standard atmospheric pressure also. Standard wall function is used in the near wall surface and adopt Reynolds-averaged Navier-Stokes equations and standard k- $\varepsilon$ model. SIMPLEC method used in the coupling of pressure and velocity. Momentum, turbulence kinetic energy and turbulence dissipation rate are second order upwind. With clear water as the medium.

According to the theory of computational fluid dynamics, the inlet of the flow uses the mass flow rate boundary condition and the outflow is used as outlet. The non-slip boundary condition is used in the solid wall, the intersection of dynamic and static regions is Interface, and adopt the multi-coordinate system[8,9].

\section{Comparative Analysis of Numerical Calculation and Experimental Results}

Formula of head:

$$
H=\frac{P_{\text {out }}-p_{\text {in }}}{\rho g}+\Delta z
$$

Where: Pout—-Total pressure of the outlet, $(\mathrm{Pa})$;

Pin-Total pressure of the imports, $(\mathrm{Pa})$;

$\Delta \mathrm{z}-$ Vertical distance between impeller inlet and guide vane outlet,(m).

Formula of efficiency calculation:

$$
\eta=\frac{\rho g q_{v} H}{M n}
$$

Where: $\mathrm{M}-$ Output torque of pump axis, $(\mathrm{N} \cdot \mathrm{m})$;

$\mathrm{N} \longrightarrow$ rotational speed,(r/min);

qv_—volume flow, (m3/s).[10]

The simulation results are calculated respectively in $0.8 \mathrm{Q}, \mathrm{Q}$ and $1.2 \mathrm{Q}$ by using the above formula, and the cure of the head compared with the test results shown in Figure.3

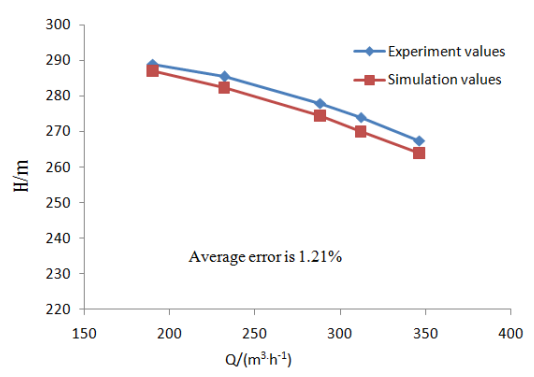

Figure 3.Comparison of Q-H cures

while the cure of the efficiency compared with the test shown in Figure.4.

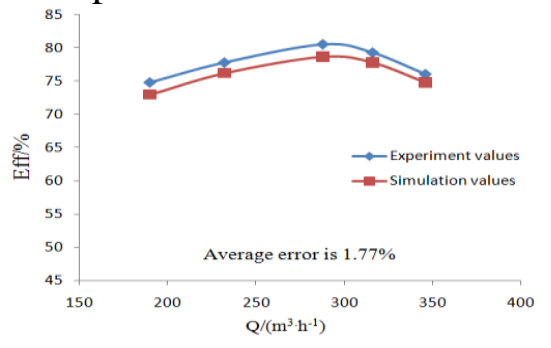

Figure 4. Comparison Q-Eff curve 
Shown as the figure. 3 and figure.4,the experiment results agree with the analysis results. There are some errors between the predictive value and the test value, leading that the results of head and efficiency are slightly higher than the simulate value. Shown by the errors contrast, the errors of both head and efficiency values are less than $2 \%$, which is allowed by numerical simulation in the range of computational error . Therefore, it is feasible to use this model to simulate the multistage centrifugal pump.

\section{Analysis of the Computation Result}

\section{The Analysis of Whole Flow Field}

The static pressure distribution of pump under design operating conditions $(80 \mathrm{~m} 3 / \mathrm{h})$ is shown in Figure.5.It can be noticed that from import to export, with the increase of the series and the working of impeller for each level, the static pressure in the flow channels at all levels shows the trend of increasing uniformity, and the static pressure reaches the maximum value at the final stage. This also shows that with the increasing of the series, the head of self-balanced multi-stage pump can be accumulated and met the delivery requirements of high lift fluid like traditional multi-stage pumps.

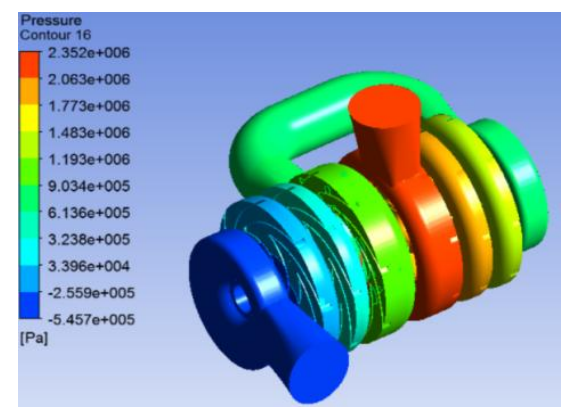

Figure5. Distribution of Static pressure of pump flow field

The vector distribution of pump under design operating conditions $(80 \mathrm{~m} 3 / \mathrm{h})$ is shown in Figure.6, The velocity field of the guide vane in different series is almost constant, and there is no obvious gradient change of the velocity between the guide vane in all series. As a cycle of deceleration, pressurize is completed after fluid flows through the every level of the guide vane of multi-stage pump, leading that the inlet velocity of the fluid is consistent between the new and the previous stage.

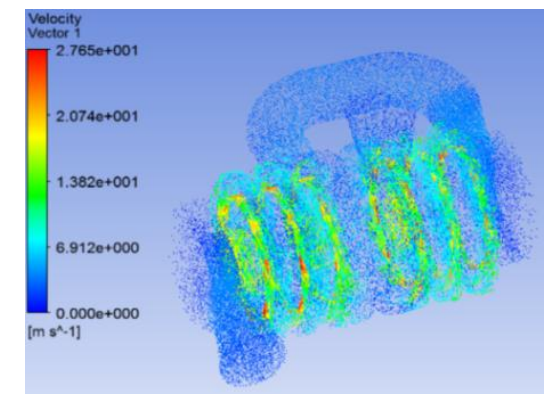

Figure 6. Distribution of vector of pump flow field

\section{The Analysis of Flow Field in First Stage Impeller and Guide Vane Median Surface}

The static pressure distribution of flow field in first stage impeller and guide vane median surface under different working conditions is shown in Figure.7.

With the increase of the flow rate, the static pressure area of the impeller is reduced, but the static pressure is basically stable from the inlet to the outlet of the impeller, while the pressure at the working face is slightly larger. The static pressure at the entrance of the guide vane is suddenly increased, mainly due to sudden expansion of the flow area when the flow of fluid from the impeller 
to guide vane inlet. With the rotation of the impeller, the relative position of impeller and the guide blade change all the time, and the flow velocity of the impeller exit is also constantly changing by the disturb of circumferential position, which resulting in pressure distribution form guide blades distributed asymmetric.
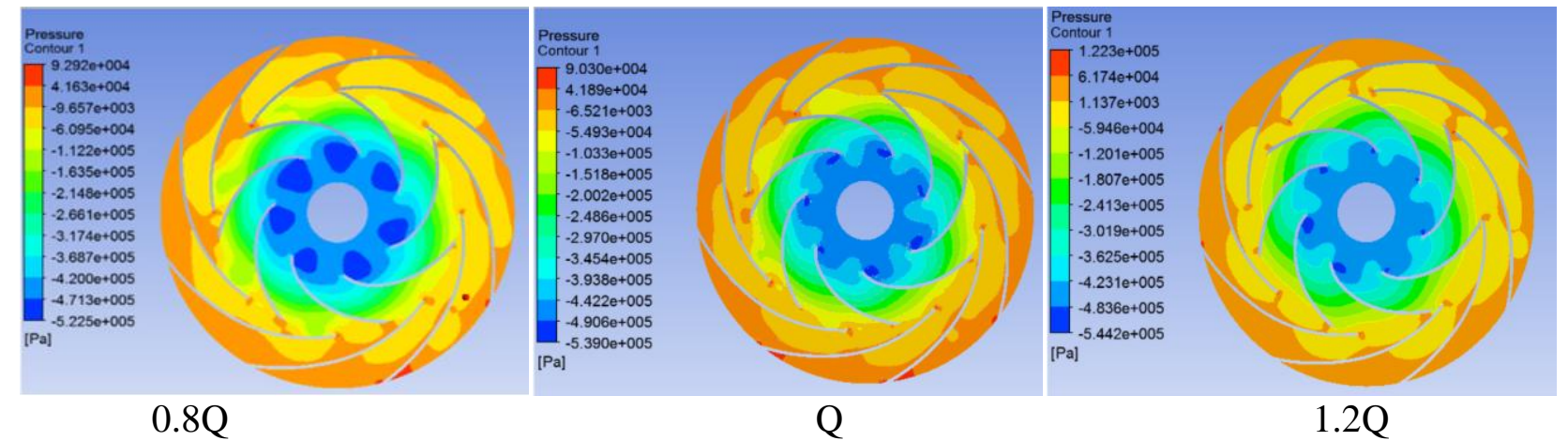

Figure.7 Distribution of Static pressure in first stage impeller and guide vane median surface

The vector distribution of flow field in first stage impeller and guide vane median surface under different working conditions is shown in Figure.8, the fluid velocity decreases with the increasing of the flow rate.

As the impeller rotation, the velocity of fluid is increased form the entrance to exit and the working surface of the impeller is significantly larger than the reverse side. When the fluid flows out of the outer edge of the impeller and into guide vane, the velocity reached the maximum. But also the fluid that empties into guide vanes will be disturbed by it, causing the flow field disordered and make loss of fluid energy, which can be seen from the non symmetry of the flow field along the circumferential direction from the inlet of the guide vane. After entering the spiral line, along the radial flow, the flow velocity at the back of the blade is less than the blade working surface. After the fluid enters the diffusion section, flow rate is further reduced as the conversion of kinetic energy into pressure energy. This is also consistent with the pressure rise in the static pressure on the previous section.

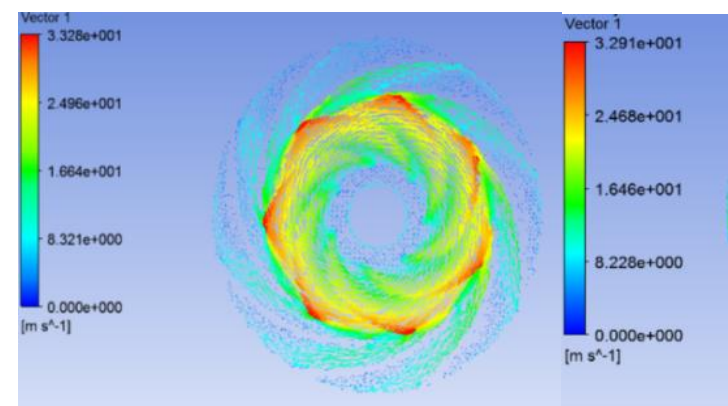

$0.8 \mathrm{Q}$

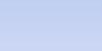

Q

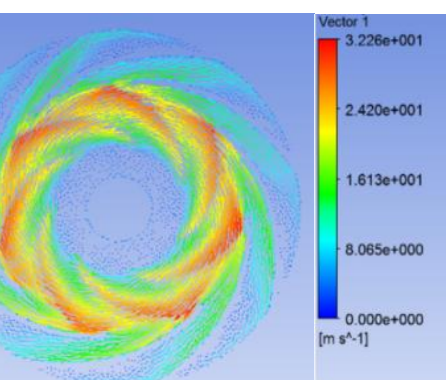

Q

(1)

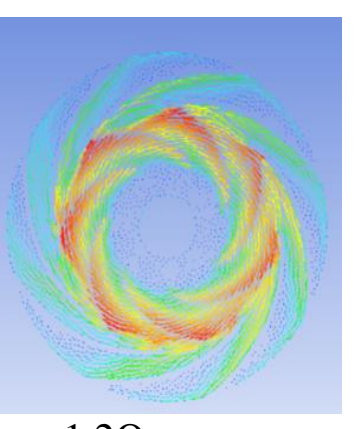

$1.2 \mathrm{Q}$

Figure.8 Distribution of vector in first stage impeller and guide vane median surface

\section{The Analysis of Flow Field in Fourth Stage Impeller and Guide Vane Median Surface}

The static pressure distribution of flow field in fourth stage impeller and guide vane median surface under three different working conditions is shown in Figure.9.

The distribution trend of static pressure in this stage impeller and guide vane medium surface as the same as the first. And with the increasing of the flow rate, the static pressure is gradually decreased under different working conditions. But the difference is that the static pressure of impeller and guide vane median surface in fourth stage is obviously larger than the first one after the working of the impeller in suction side. 


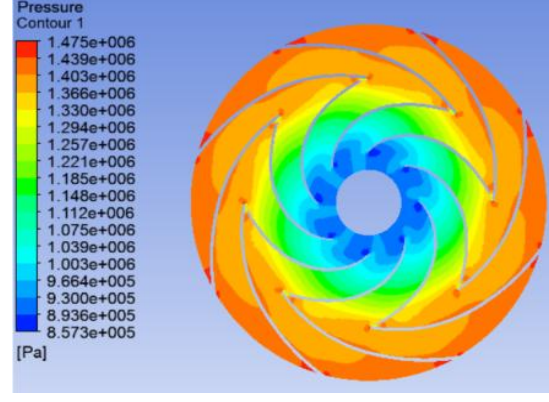

$0.8 \mathrm{Q}$

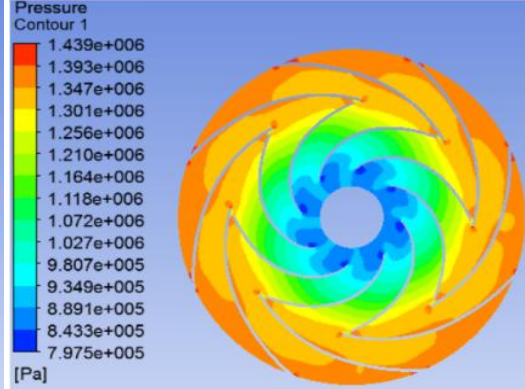

Q

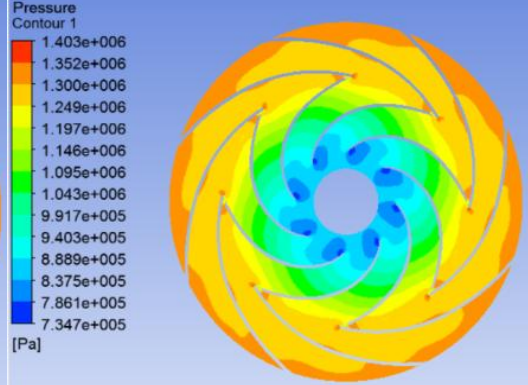

$1.2 \mathrm{Q}$

Figure.9 Distribution of Static pressure in fourth stage impeller and guide vane median surface

The vector distribution of flow field in fourth stage impeller and guide vane median surface under different working conditions is shown in Figure.10.

As the same as the first stage, with the impeller rotation, the velocity of fluid is increasing from the entrance to exit and the working surface of the impeller is significantly larger than the reverse side. When the fluid flows out of the outer edge of the impeller and gets into guide vane the velocity reaches the maximum. The distribution trend of vector in this stage impeller and guide vane median surface as the same as the first. However, when the fluid flows through the pipe and the second stage suction, the complex motion causes the fluid energy loss. Therefore, the flow rate is lower than first stage after a complex flow in the pipe and the secondary suction section.

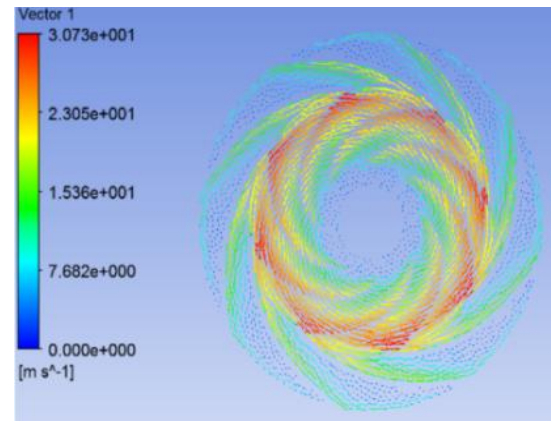

$0.8 \mathrm{Q}$

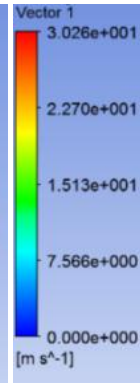

$-1]$

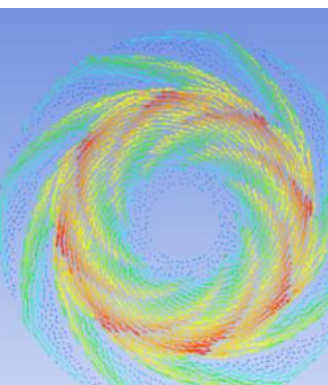

Q

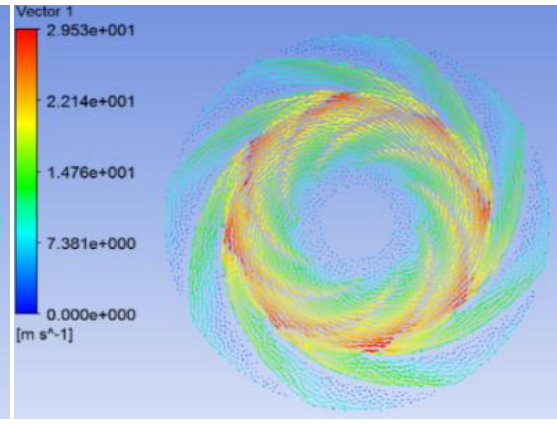

$1.2 \mathrm{Q}$

Figure.10 Distribution of vector in fourth stage impeller and guide vane median surface

\section{Conclusions}

(1)With the increasing of the flow rate, the head of the multi-stage pump is decreasing under simulated conditions. The efficiency curve is increased first and then decreased and the maximum value is obtained at the point of the rated condition.

(2)The distribution trend of impeller and guide vane in the first and fourth stage is consistent. Shown as the figure of static pressure, the impeller and guide vane in fourth stage is obviously larger than the first. Shown as the figure of vector, the flow rate of fourth stage is lower than first stage after a complex flow in the pipe and the secondary suction section.

(3)The predicted hydraulic performance curve is basically consistent with the actual hydraulic performance curve. It is proved that the reliability of the numerical simulation of self-balancing multistage pump by using the standard model, which has certain guiding significance for the design and optimization of self balanced multi-stage centrifugal pump.

\section{Acknowledgements}

This project is supported by Major Projects of Hunan Province Science and Technology Plan(2014FJ1002) and the key project of Science Research of Hunan Provincial Department of Education(Grant No.15A185). 


\section{References}

1. Gantar Mar jan, Florjancic Dusan, Sirok Brane. Hydraulic axial thrust in multistage pumps origins and solutions[J]. J. Fluids Eng. Trans. ASME, 124(2): 336-341(2002)

2. Huang Si, Wang Guoyu.Analysis of flow field asymmetry and force on centrifugal pump by 3-Dnumerical simulation[J]. Transactions of the Chinese Society for Agricultural Machinery, 37(10): 66-69(2006)

3. Huang Jianfeng, Zhang Lixiang, Wang Wenquan, et al. Fine simulation of 3-D unsteady flows in a Francis hydro-turbine on detached eddy simulation[J]. Proceedings of theCSEE,31(26):83-89(2011)

4. Kong Fanyu, Liu Jianrui, Shi Weidong, et al. Calculation of axial force balance for high- speed magnetic drive pump[J]. Transactions of the Chinese Society of Agricultural Engineering (Transactions of the CSAE),21(7):69-72(2005)

5. Huang Si,Deng Qingjian,Wang Hongjun. 3-D analysis for transient flow in a multistage centrifugal pump using sliding mesh [J]. Fluid Machinery,37(3):24-27(2009)

6. Wang Jiaqiong, Kong Fanyu. Study on the optimization of the hydraulic performance of the impeller and the guide vane of the multistage centrifugal pump[J].Journal of Huazhong University of Science and Technology (Natural Science Edition),03:92-96(2013)

7. Shi Weidong, Li Qifeng, Lu Weigang, et al. Estimation and experiment of axial thrust in centrifugal pump based on CFD $[\mathrm{J}]$. Transactions of the Chinese Society for Agricultural Machinery, 40(1): 60-63(2009)

8. Wang Yang, Zhao Lifeng, Liu Zhichao, et al. Numerical prediction of the hydraulic performance of multi stage centrifugal pump by vorticity analysis method[J].Journal of irrigation and drainage engineering.07:561-566(2016)

9. Liu Yuanyi ,Wang Guangye. Computer aided analysis of the internal flow field of the stamping and welding multistage centrifugal pump impeller[J]. Journal of Mechanical Engineering. 08:207-211(2007)

10. Zhang Xuejing, Li Deming.Prediction of performance of multi stage centrifugal pump by CFD numerical calculation[J]. Pump technology. 05:30-33(2005) 